\title{
Document Image Retrieval Based on Visual Saliency Maps
}

\author{
Fahimeh Alaei \\ School of ICT, Griffith \\ University, Australia \\ fahimeh.alaei@griffithuni.edu.au
}

\author{
Alireza Alaei \\ Southern Cross University, \\ Australia \\ alireza20alaei@gmail.com
}

\author{
Umapada Pal \\ CVPR Unit, Indian \\ Statistical Institute, India \\ umapada@isical.ac.in
}

\author{
Michael Blumenstein \\ University of Technology \\ Sydney, Australia \\ Michael.Blumenstein@uts.edu.au
}

\begin{abstract}
There has been a massive growth in the production of various unstructured, complex and multi-lingual digitised documents in recent years. Storing and manipulating such digitised documents towards a paperless society has been the objective of emerging technology. As the human visual system can easily distinguish the global summary of images, extracting features based on human attention from images is desirable to achieve more accurate document image retrieval results. Thus, in this research work, an appearance-based document image retrieval system using image saliency maps depending on human visual attention is proposed. The saliency map obtained from the input document image is used to generate a weighted document image. Features are then extracted from the weighted document images using the Gist operator. Then, locality-sensitive hashing is considered to compute similarity distances between a query and the document images in the knowledge-based database. To evaluate the performance of the proposed document image retrieval system MTDB, ITESOFT, and CLEF-IP datasets of document images were used for experimentation. The proposed document image retrieval system provided promising retrieval results compared to the results reported in the literature.
\end{abstract}

Keywords: document image retrieval; visual saliency map; Gist operator

\section{INTRODUCTION}

The impetus of current technology is towards a paperless world. As a common organisational practice and for economic feasibility, an enormous number of digitised paper-based documents including books, letters, historical manuscripts, official letters, cheques, etc. are usually generated or captured through electronic devices, such as scanners, fax machines, digital cameras, and mobile phones in everyday life, and then saved on giant hard disks or in the cloud for later use. These large quantities of digitised document images have, however, been generated and saved without any labeling or sufficient indexing information. Automatic extraction, classification, clustering, and searching for information from such a large amount of data is a challenging task.

In recognition-based document image retrieval (DIR) systems, a traditional technique, optical character recognition (OCR), is used to convert document images to a machinereadable text format to enable document retrieval processing. However, it should be noted that OCR may not always be a practical solution for dealing with such a massive volume of document images, especially when it is applied on document images which are complex in layout, degraded and of poor quality [1]. To overcome some of those difficulties in document image retrieval, many recognition-free document image retrieval techniques using logo, trademark, handwritten signature, and shape coding methods have been developed to provide an effective way of retrieving and organising document images [2-5]. Content-specific features, bag-ofwords features, key-region detection, and layout-specific structures have also been considered in the literature for the document image retrieval process $[4,6]$.

Texture feature analysis has played a significant role in different research areas, such as medical imaging, industrial inspection, and remote sensing $[9,10]$. A repetitive pattern of information or arrangement of a structure with orderly intervals is defined as a texture. This also refers to the appearance of an object given by shape, size, density, and arrangement of regions within an image [7,9]. A texture-based feature extraction method basically collects such features/characteristics through a texture analysis process. Texture features are generally fast and simple to compute and they are computationally suitable for large volumes of data. Many researchers from the document image analysis (DIA) community have also used various texture features in different stages of DIA systems, including segmentation, layout analysis, recognition [11,12], etc. Furthermore, texture features have been used to discriminate textual and nontextual areas, and to differentiate between various types of text fonts and graphics in document images [13].

Alternatively, appearance-based features have traditionally been used in the field of computer vision and image processing for natural scene image analysis $[14,15]$. In [16], Gist features and saliency maps have been considered for detecting and classifying targets in high-resolution satellite images. The method relies on extracting statistical signatures of targets. To do so, the input image was divided into small blocks and two sets of features; saliency and Gist were extracted to achieve the classification results. In [17], Gist features with angular radial partitioning were used for natural scene classification. The angular partitioning was considered to capture finer details of scene images. In [18], bottom-up and top-down saliency detection approaches have been considered for image retrieval. The distinctiveness score in the saliency map of each image has been calculated in a hierarchical manner and used to re-rank the images. In [19], a saliencybased feature extraction method was used for semantic scene image retrieval. Saliency features have first been used to segment images in order to separate the background region and foreground objects. Then, the scale invariant feature transform (SIFT) features were extracted from both background and foreground regions of the images for the retrieval process.

As little attention has been paid to appearance-based texture features for document image retrieval, the main focus of this research work is to propose appearance-based texturebased features for document image retrieval, providing a new method similar to human perception for image retrieval. The present research work explores, for the first time, a document image retrieval process by using saliency maps to give higher weight to the foreground of document images. Features are 
extracted from weighted document images through the Gist descriptor. In the proposed DIR system, knowledge-based features are then created using the training samples and locality-sensitive hashing is finally employed to compute similarity distances between a given query and document images in the database to provide retrieval results. In the proposed system, features are extracted from salient regions, which have a particular sign of attraction for human observers.

The rest of the paper is organized as follows. In Section II, the proposed document image retrieval approach is explained. Section III discusses experimental results and comparative analysis. Finally, conclusions are presented in Section IV.

\section{PROPOSED METHOD}

Visual attention provides the ability to quickly pick up the most important information from an image [20]. The regions of an image that attract the greatest attention from the human visual system or have a particular sign of attraction for a human observer are called salient regions. In the literature, great efforts have been dedicated to generate the saliency maps of images without means of eye-trackers and human involvement [20, 21]. Saliency detection has been considered for different applications, such as image segmentation, object detection, object recognition, and content-based image retrieval (CBIR) [21]. As saliency regions attract superior attention through the visual system, they can provide valuable features for retrieval tasks. In this research work, a DIR method based on human visual attention is proposed. The block diagram of the proposed method is shown in Fig. 1. In the proposed system, the saliency map of the input document image is initially obtained to form a weighted document image, combining the saliency map and the original document image. Feature extraction is then employed on the weighted document image to create a knowledge-based model for training images. The main and new contribution in the proposed method here is the use of human visual attention for document image retrieval.

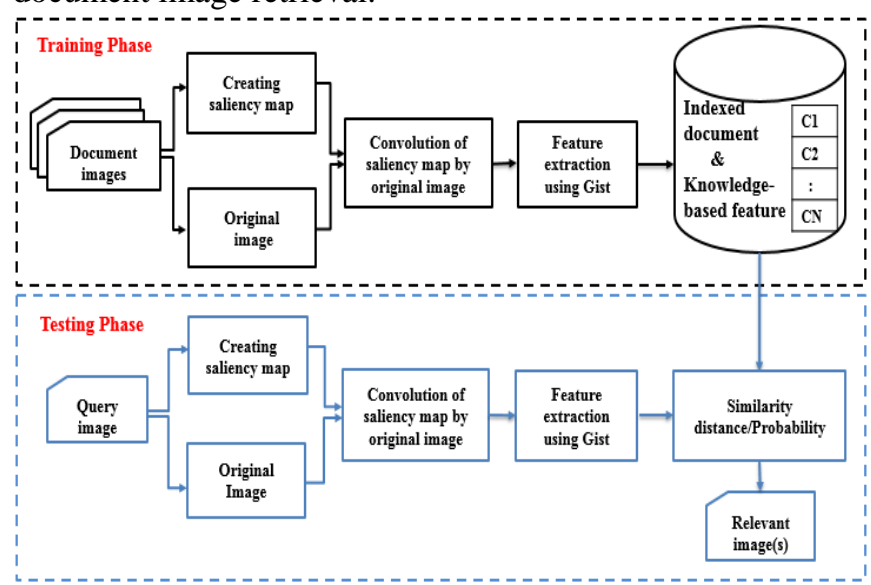

Fig. 1. Block diagram of the proposed method

\section{A. Feature Extraction}

\section{Visual Saliency Map Generation}

Saliency estimation approach in the literature can be categorised into two different approaches: top-down and bottom-up. In top-down models, high-level signs, such as context, semantic, and knowledge information, are used to compute a saliency map. However, bottom-up models are designed based on low-level local features, such as colour, contrast, texture, intensity, motion, and orientation [21, 22].

Bottom-up visual saliency detection models have provided outstanding results compared to the human visual system. Visual saliency has been used in the literature in different applications [19]. However, to the best of our knowledge, no document image retrieval method in the literature has incorporated visual saliency map detection in the document image retrieval pipeline. In this research work, therefore, one of the best state-of-the-art bottom-up methods, the hypercomplex Fourier transform (HFT), as a transform-based approach [20], is considered to create a visual saliency map for a given document image to be further used for DIR. Transform-based approaches have provided better DIR results in the literature [24]. Thus, the HFT saliency detection method, which is also a transform-based technique, is considered for generating visual saliency maps in the proposed DIR method. To get an idea of the HFT model, a brief description of the HFT is provided in the following.

The hyper-complex Fourier transform has been considered to combine multiple feature maps for extracting saliency maps [20]. The input of a hyper-complex matrix is specified to be a quaternion, represented as follows:

$$
f(n, m)=a+b_{i}+c_{j}+d_{k}
$$

where $a, b, c$, and $d$ are real numbers and $i^{2}=j^{2}=k^{2}=$ $i j k=-1$. The discrete version of the hyper-complex Fourier transform can be represented as:

$$
F_{H}[u, v]=\frac{1}{\sqrt{N M}} \sum_{n=0}^{N-1} \sum_{m=0}^{M-1} e^{-\rho 2 \pi\left(\left(\frac{n u}{N}\right)+\left(\frac{m v}{M}\right)\right)} f(n, m)
$$

The inverse of hyper-complex Fourier transform is defined as:

$$
f(n, m)=\frac{1}{\sqrt{N M}} \sum_{u=0}^{N-1} \sum_{v=0}^{M-1} e^{\rho 2 \pi\left(\left(\frac{n u}{N}\right)+\left(\frac{m v}{M}\right)\right)} F_{H}[u, v]
$$

where $\rho$ is a unit pure quaternion and $\rho^{2}=-1$. In [20], the input of hyper-complex matrix is described as:

$$
f(n, m)=w_{1} f_{1}+w_{2} f_{2} i+w_{3} f_{3} j+w_{4} f_{4} k
$$

where $w$ and $f$ stand for weight and feature map, respectively. To compute the saliency for the static input case, three features including $f_{2}=(r+g+b) / 3, f_{3}=R-G$, and $f_{4}=B-$ $Y$ have been considered. Three channels of an input image, red, green, and blue, are shown by $r, g$, and $b: R=r-$ $(g+b) / 2, G=g-(r+b) / 2, B=b-(r+g) / 2$. The colour space representation of the input image is used in these three feature maps. In equation (4), $f_{1}$ has been considered for the videos, and for static images to the value of $w_{1}=0$.

To compute the saliency map, the hyper-complex Fourier transform is revised and written in polar form as follows:

$$
F_{H}[u, v]=\left\|F_{H}[u, v]\right\| e^{\rho \Phi}(u, v)
$$

where $\|$.$\| points to the modulus for each element of a$ hypercomplex matrix, and $F_{H}[u, v]$ are considered as the frequency domain representation of $f(n, m)$. The amplitude spectrum $A(u, v)$, phase spectrum $p(u, v)$, and eigenaxis spectrum $X(u, v)$ are defined as follows:

$$
\begin{gathered}
A(u, v)=\left\|F_{H}[u, v]\right\|, \\
p(u, v)=\Phi(u, v)=\tan ^{-1} \frac{\|v(F(u, v))\|}{s(F(u, v))}
\end{gathered}
$$




$$
X(u, v)=\rho(u, v)=\tan ^{-1} \frac{v(F(u, v))}{\|v(F(u, v))\|}
$$

The spectrum scale space $\Lambda=\left\{\Lambda_{k}\right\}$ is created by smoothing $A(u, v)$. It should be noted that information contained in the amplitude plots is dependent on scale $k$. The inverse transform is performed to provide a saliency map at each scale as follows:

$$
S_{k}=g *\left\|F_{H}^{-1}\left\{\Lambda_{k}(u, v) e^{x p(u, v)}\right\}\right\|^{2}
$$

where $g$ is a Gaussian kernel at a fixed scale. At this stage, a series of saliency maps $\left\{S_{k}\right\}$ is created to get the best saliency map from the series of saliency maps. The principle for determining the ideal scale is based on the following:

$$
k_{p}=\arg \min _{k}\left\{H\left(S_{k}\right)\right\}
$$

In the hyper-complex Fourier transform, a parameter $\gamma$ has been considered for each candidate saliency map:

$$
\gamma_{k}=\sum \sum K(n, m) \cdot N\left(S_{k}(n, m)\right),
$$

where a 2D-centered Gaussian mask is shown by $K$ and is the same size as $S$. The equation $\sum \sum K(n, m)=1 . N($.$) is$ considered for normalizing $S$, therefore, the summation of all the pixel values is 1 . Finally, $k_{p}$ can be rewritten as:

$$
k_{p}=\arg \min _{k}\left\{\gamma_{k}^{-1} H_{2 D}\left(S_{k}\right)\right\} \text {. }
$$

\section{Weighted Document Image}

Based on the HFT method described in the previous subsection, a saliency map which displays significant information of the input document image, is created for each document image. To incorporate the visual saliency map obtained from the input document image, a weighting strategy is proposed in this research work. The weighting process can be applied at different levels (e.g., document and feature). In this research work, the weighting is applied at the document level. To give a weight to each pixel in the document image, the created saliency map of the image is convolved by the original document image. To formularise the process, the weighted document image can be computed as follows:

$$
W(n, m)=I(n, m) \cdot S_{k}
$$

where $I$ is the input document image of size $N \times M$ and $S_{k}$ is the best saliency map obtained from the input document image I. A sample original document image, the extracted visual saliency map of the document image, and the weighted document image, which constitute the weighting process, are shown in Fig. 2.

\section{Gist Descriptor}

It is well established from a variety of studies that the Gist operator can provide promising retrieval performance [23, 24]. In addition, the Gist operator is an appearance-based feature extraction method, which provides meaningful information from an image at a glance. Thus, Gist features are selected to extract salient information from weighted document images created by convolution of the saliency map and the original document image.

The Gist descriptor was initially introduced to provide a low-dimensional representation of the structure of images [14]. The spatial structure of the image is presented by naturalness, roughness, ruggedness, openness, and expansion as perceptual dimensions. The Gist descriptor has been used for other applications, such as scene detection and classification in the literature $[16,17,25]$. The Gist descriptor simply uses low-level features to compute higher-level semantic properties and usually consists of the semantic label and the spatial layout of images.

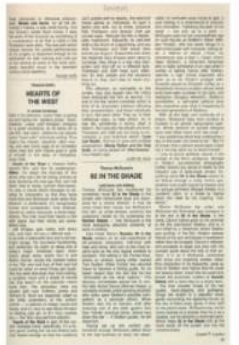

(a)

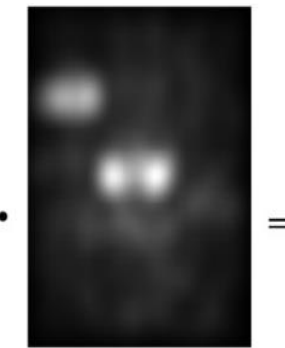

(b)

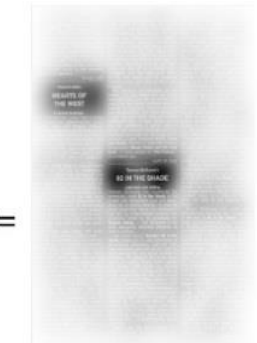

(c)
Fig 2. Example of giving weight to the document image: (a) the original document (b) extracted saliency map, and (c) weighted document image.

Supplementary information of an image, which is also useful for the retrieval process, can be extracted from different scales of the image. The input weighted image $W$ of size $N \times M$, which is a grey level image, can be decomposed into different scales.

$$
W_{q}(n, m)=(\downarrow 2) p_{q-1}(n, m), q=0, \ldots, Q
$$

where $(\downarrow 2)$ shows the down-sampling operation and $Q$ shows a spatial scale. Gaussian pre-filtering is applied on the weighted image to prevent the local image regions being dominated by the energy spectrum. After pre-filtering, the Fourier transform is applied on different scales of the weighted image, which corresponds to a spatial distribution of spectral information $W_{q}(n, m)$ and is presented as follows:

$$
W_{q}\left(f_{n}, f_{m}\right)=\sum_{n=0}^{N-1} \sum_{m=0}^{M-1} W_{q}(n, m) h(n, m) e^{-j 2 \pi\left(f_{n} n+f_{m} m\right)}(15)
$$
where spatial frequency variables are denoted by $f_{n}$ and $f_{m}$, and a circular Hanning window, which reduces boundary affects, is represented by $h(n, m)$. The spatial frequencies of the image obtained from the energy spectrum $E_{q}\left(f_{n}, f_{m}\right)^{2}=$ $\left|W_{q}\left(f_{n}, f_{m}\right)\right|^{2}$, provides structural information. The information obtained is about the orientation, smoothness, length and width of the image. Then, a Gabor filter is applied on the image to generate spectrum maps sampled with coarse resolution. The maps are generated at four different angles $\left(\theta_{i}=0,45,90,135\right)$ and at eight spatial scales $(c=$ $0,1,2, \ldots, 7)$, which can be defined as $M_{i}(c)=\operatorname{Gabor}\left(\theta_{i}, c\right)$.

The energy spectrum $E_{q}\left(f_{n}, f_{m}\right)^{2}$ is then represented by a series of maps. Each one of these maps represents one orientation in one scale of the weighted image. Each map, which is of size $256 \times 256$, is decomposed into 16 fixed grids of $64 \times 64$. The mean value is calculated in each grid to compute 16 raw gist features $G_{i}^{k, l}(c, q)$ per map:

$$
G_{i}^{d, z}(c, q)=\frac{1}{16 B H} \sum_{u=\frac{d B}{4}}^{\frac{(d+1) B}{4}-1} \sum_{v=\frac{z Y}{4}}^{\frac{(z+1) Y}{4}-1}\left\lfloor M_{i}(c, q)\right\rfloor(u, v)
$$

where $d$ and $z$ are the horizontal and vertical direction, and $B$ and $Y$ are the sizes of the map. As a result, 16 mean values of all 32 feature maps are linearly combined to produce a Gist feature set of size $16 \times 32=512$ features . 


\section{B. Creation of a Knowledge-based Feature}

Gist features extracted from each image in the training phase are kept/saved as a knowledge-based database for the testing process. The size of the knowledge-based database is dependent on the number of training samples and the number of features (feature size). The former indicates the number of rows, whereas the latter demonstrates the number of columns in the database.

\section{Similarity Matching}

In the testing phase of the proposed method, first, the proposed visual saliency map detection method is employed on the image to extract the saliency map of the given query. Then, employing the weighting process on the original image and saliency map, a weighted document image is obtained. The Gist feature extraction method is applied to the weighted image to characterise the given query. Similarity distances between the extracted features from the query image and the knowledge-based feature vectors are computed and the document images are finally ranked based on similarity scores. The document image(s) with a maximum visual similarity to the query image are retrieved accordingly.

To have a fast and effective retrieval system, a fast measuring distance is necessary. In the previous studies, the $\mathrm{k}$-nearest neighbor ( $\mathrm{k}-\mathrm{NN}$ ) method has usually been used to find the similarity distance between a query image and trained document images $[10,11]$. Since we have a large dataset, locality-sensitive hashing (LSH) [13] is taken into account in this work. In LSH, time complexity is highly reduced, due to the creation of a group of buckets, which contain smaller data elements, compared to the k-NN methods.

\section{Locality-sensitive Hashing}

The locality-sensitive hashing (LSH) method is a similarity search method, which is insensitive to the dimensionality of datasets and has linear computational complexity. For large datasets of high dimension, localitysensitive hashing [13] is the most valuable method using probability distributions to retrieve similar images in relation to a given query.

By letting $e$ represent a dataset and $t$ a metric, in a metric space $(e, t)$, a set of hash functions $\left(h_{1}, \ldots, h_{n}\right)$ is randomly selected from a family $H$ for adjusting the LSH. In the view of $P(A)$ as a probability of event $A$, the function family $H$ is an $\left(c, r, p_{1}, p_{2}\right) \mathrm{LSH}$ family. The equation (17) is satisfied by any $h_{i} \epsilon H$, if and only if, $r$ is a positive value, $c$ is a real number, $c>1$ and $x, y \in e$.

$$
\left\{\begin{array}{l}
p\left(h_{i}(x)=h_{i}(y)\right) \geq p_{1}, \quad \text { if } t(x, y) \leq r \\
p\left(h_{i}(x)=h_{i}(y)\right) \leq p_{2}, \quad \text { if } t(x, y)>c \times r
\end{array}\right.
$$

where $p_{1}>p_{2}$

The LSH method hashes all the points in the dataset $e$ to index the data points in $e$ with a hash function. If $x, y \in e$ are close together by definition of the metric $t$, then $k_{x}$ and $k_{y}$ are identical. In contrast, if $x, y \in e$ are far from each other, the hash keys show a low probability of being the same. The data points which have the same hash key are located in a connecting area of the physical memory and the hash key is used to address the hash bucket. To have an orderly save of the hash keys, a hash table is used [26]. When more than one set of hash functions is generated, LSH usually uses more than one hash table. Thus, $p_{1}$ increases and $p_{2}$ decreases. Giving a query point, LSH retrieves all the data points from the hash bucket to find the nearest point to a given query image.

The similarity between a given query and all the trained documents are computed using the LSH method. The document images with the highest similarity to a given query are listed as the Top-n. Based on the mean $(\mu)$ and standard deviation $(\sigma)$ of the distance matrix obtained from the trained samples, a threshold $\tau=\mu+\gamma \times \sigma$ is calculated, where $\gamma$ is a tuning parameter. The threshold $\tau$ is considered to accept or reject the ranked Top-n document images in a class. In our experiment, different small values between 0 and 1 were considered to get the best retrieval results (F-score) from the training samples. This fixed value of $\gamma$ was used in the testing phase experimentation.

\section{EXPERIMENTAL ANALYSIS}

\section{A. Databases and Evaluation Metrics}

Three datasets consisting of high dimensional images with a great diversity of page layouts were considered for evaluation of the proposed method.

The first database is the Media Team Document Database (MTDB) from the University of Oulu, which contains various types of scanned documents [27]. The MTDB is an unbalanced dataset, as different numbers of samples exist in each class and includes three different resolutions; the size of high-resolution document images in MTDB is approximately $2800 \times 1700$ pixels. Eleven classes among 19 classes of the MTDB that contain mainly text documents were considered for the experiments in this research work. In total, 1,322 document samples were used for the experimentation.

The second database is the ITESOFT dataset [28]. The ITESOFT database is a collection of several scanned official documents from 12 different classes. The ITESOFT dataset is also an unbalanced dataset and includes two different resolutions. High-resolution document images considered for our experiments are of $3700 \times 2500$ pixel size. In total, 1,116 document samples from the ITESOFT dataset were used for the experimentation.

The CLEF-IP dataset is also a collection of heterogeneous document images composed of 9 different classes [29]. The flowchart class includes only a few samples, so it was not considered in our experiments. The dataset contains a large variety of document images of sizes from $35 \times 47$ pixels to $2000 \times 2500$ pixels. In total, 37,771 document samples were used for experimentation.

For the evaluation metrics, the precision $(P)$, recall $(R)$ and F-score, as three commonly used metrics in the image retrieval literature, were considered in this research work. The number of correctly retrieved document images over the number of retrieved documents is defined as $P$, and the number of correctly retrieved documents over the actual number of document images is defined as $R$. The F-score is derived from the precision and recall and is defined as $F=2 \times(P \times R)$ / $(P+R)$. Since the F-score reflects the degree of balance between precision and recall, instead of only the absolute value of the precision and recall, it is a precise measurement.

The F-scores were measured according to the maximum visual similarity to a given query that placed first (Top-1), Top-3, Top-5, and Top-10 of the ranked list. The Top-1 value 
indicates the percentage of correct retrieval results at the first position with respect to a given query. Subsequently, the Top10 value represented the performance of the system when the document image retrieval system could rank the correct document image with respect to a given query in the first 10 places in the ranked list of document images.

A cross-validation technique was conducted in our experiments on each dataset to randomly select $33 \%$ of the samples from each class in each round for training, and the rest of the samples for testing. The training and testing sets did not have any overlapping in the experiments. To evaluate the performance of the proposed method, the experiments were repeated 30 times to remove any inconsistency from the reported results. As final results, the mean $P$, mean $R$ and mean F-score were calculated and reported for the proposed method on each dataset.

It should be noted that the significance of resampling in the retrieval results was also checked by employing the oneway ANOVA test on the F-scores obtained. A null hypothesis against the alternative hypothesis was considered for testing purposes. In the results, the null hypothesis was not rejected as we obtained $p>0.05$ for all three datasets. Thus, resampling in each run did not have a significant influence on the F-scores.

\section{B. Results and Discussion}

The results obtained from our proposed method on three datasets are demonstrated in Table I. By applying the proposed method on MTDB, $79.28 \%$ and $91.73 \%$ of the document images were retrieved correctly at Top-1 and Top-10 choices, respectively. Similarly, the proposed method respectively provides $91.10 \%$ and $97.68 \%$ F-scores in the Top-1 and Top10 choices on the ITESOFT dataset. By applying the proposed method on the CLEF-IP dataset, $82.32 \%$ and $98.90 \%$ F-scores in the Top-1 and Top-10 choices were obtained for document image retrieval.

TABLE I. THE RETRIEVAL RESULTS OBTAINED FROM THE PROPOSED METHOD ON THREE DATASETS.

\begin{tabular}{llcccc}
\hline Dataset & $\begin{array}{l}\text { Similarity } \\
\text { distance }\end{array}$ & $\begin{array}{c}\text { Top-1 } \\
(\mathbf{\%})\end{array}$ & $\begin{array}{c}\text { Top-3 } \\
(\mathbf{\%})\end{array}$ & $\begin{array}{c}\text { Top-5 } \\
(\mathbf{\%})\end{array}$ & $\begin{array}{c}\text { Top-10 } \\
(\mathbf{\%})\end{array}$ \\
\hline MTDB & Precision & 66.78 & 77.10 & 80.63 & 84.94 \\
& Recall & 97.54 & 97.68 & 98.46 & 99.70 \\
& F-score & $\mathbf{7 9 . 2 8}$ & $\mathbf{8 6 . 1 8}$ & $\mathbf{8 8 . 6 6}$ & $\mathbf{9 1 . 7 3}$ \\
\hline ITESOFT & Precision & 83.70 & 90.18 & 92.61 & 95.47 \\
& Recall & 99.94 & 99.94 & 100 & 100 \\
& F-score & $\mathbf{9 1 . 1 0}$ & $\mathbf{9 4 . 8 1}$ & $\mathbf{9 6 . 1 6}$ & $\mathbf{9 7 . 6 8}$ \\
\hline \multirow{3}{*}{ CLEF-IP } & Precision & 69.95 & 86.39 & 91.13 & 97.83 \\
& Recall & 100 & 100 & 100 & 100 \\
& F-score & $\mathbf{8 2 . 3 2}$ & $\mathbf{9 2 . 7 0}$ & $\mathbf{9 5 . 3 6}$ & $\mathbf{9 8 . 9 0}$ \\
\hline
\end{tabular}

To examine the robustness of the proposed system in relation to noisy documents, a set of experiments was conducted by applying the Gaussian noise with different variances ranging from 0.01 to 0.05 on the document images. It is worth noting that by applying noise with variances of 0.01 , and 0.02 on document images, no remarkable changes have appeared in the results. However, the performance of the system has dropped up to $2 \%$ when we applied the Gaussian noise with 0.05 variance on document images from different datasets.

TABLE II. THE RETRIEVAL RESULTS OBTAINED FROM THE GIST OPERATOR ON THREE DATASETS.

\begin{tabular}{llcccc}
\hline Dataset & $\begin{array}{l}\text { Similarity } \\
\text { distance }\end{array}$ & $\begin{array}{c}\text { Top-1 } \\
(\mathbf{\%})\end{array}$ & $\begin{array}{c}\text { Top-3 } \\
(\boldsymbol{\%})\end{array}$ & $\begin{array}{c}\text { Top-5 } \\
(\mathbf{\%})\end{array}$ & $\begin{array}{c}\text { Top-10 } \\
(\boldsymbol{\%})\end{array}$ \\
\hline MTDB & Precision & 59.26 & 69.13 & 72.96 & 78.91 \\
& Recall & 99.85 & 100 & 100 & 100 \\
& F-score & $\mathbf{7 4 . 3 7}$ & $\mathbf{8 1 . 7 4}$ & $\mathbf{8 4 . 3 7}$ & $\mathbf{8 8 . 2 1}$ \\
\hline ITESOFT & Precision & 74.56 & 85.45 & 87.56 & 91.44 \\
& Recall & 99.79 & 99.97 & 100 & 100 \\
& F-score & $\mathbf{8 5 . 3 5}$ & $\mathbf{9 2 . 1 4}$ & $\mathbf{9 3 . 3 7}$ & $\mathbf{9 5 . 5 3}$ \\
\hline \multirow{3}{*}{ CLEF-IP } & Precision & 67.89 & 83.71 & 88.56 & 93.24 \\
& Recall & 99.99 & 100 & 100 & 100 \\
& F-score & $\mathbf{8 0 . 8 7}$ & $\mathbf{9 1 . 1 3}$ & $\mathbf{9 3 . 9 3}$ & $\mathbf{9 6 . 5 0}$ \\
\hline
\end{tabular}

To demonstrate the significance of our proposed method for document image retrieval compared to the original Gist operator, the results obtained using only the Gist operator on the same datasets are illustrated in Table II. From Table I and Table II, it is apparent that the proposed method integrating saliency map detection following Gist feature extraction provided higher retrieval F-scores compared to the method using the Gist operator alone for feature extraction. In the MTDB dataset, our proposed method shows up to 5\% improvement in F-scores at different levels. Similarly, the proposed method provided approximately $6 \%$ and $2 \%$ better F-score results respectively, on the ITESOFT and CLEF-IP datasets. It is noticeable that when using the appearance-based texture feature, the retrieval results can be significantly improved by assigning a higher weight to the foreground of the document images using saliency maps.

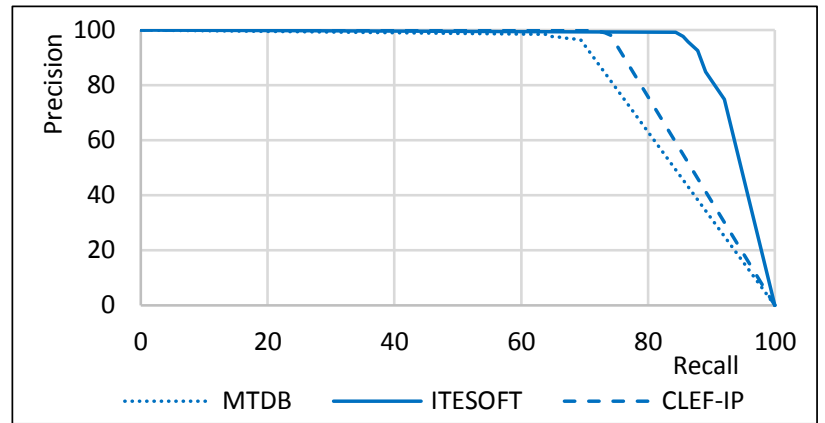

Fig 3. The precision-recall curve for the proposed method on three datasets.

Fig. 3 shows the precision-recall curve for the proposed method on three datasets. The proposed method provided better results on the ITESOFT dataset compared to the other two datasets.

\section{A. Comparative Analysis}

The results obtained from the proposed method are compared with the results obtained from the state-of-the-art methods on three datasets, and a comparison of the results is shown in Fig. 4. The methods that used a combination of the Gist and wavelet transform features [23], as well as the LBP and wavelet transform features [11] for document image 
retrieval, were considered for comparison. As demonstrated in Fig. 4, the results (especially Top-1 results) obtained from the proposed method outperformed the state-of-the-art results on three datasets.

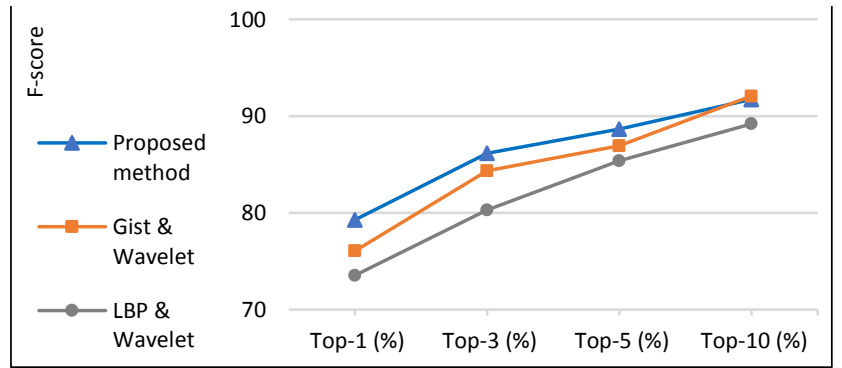

(a)

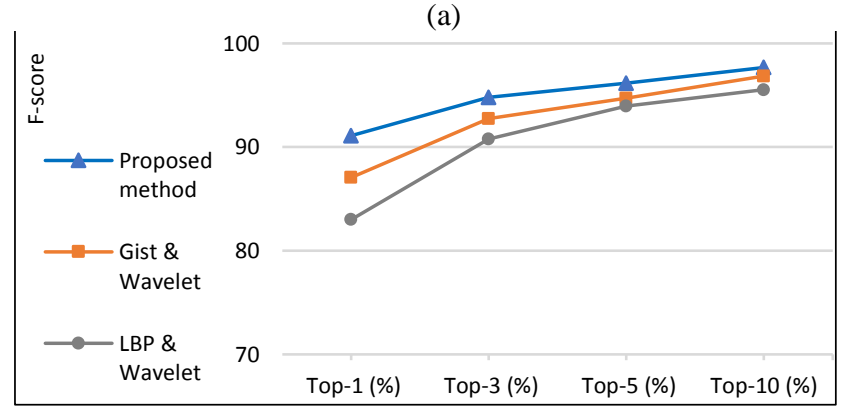

(b)

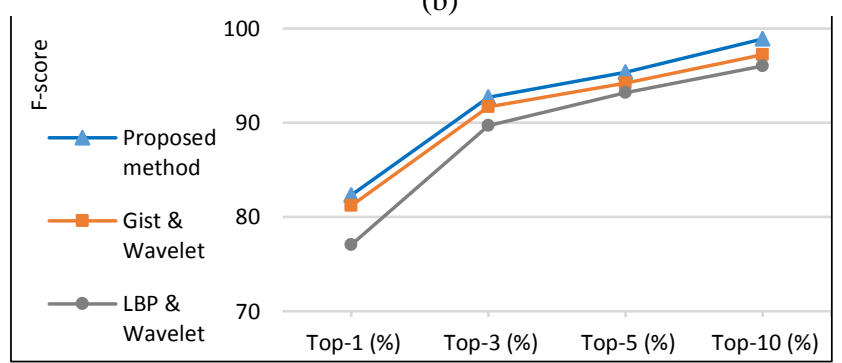

(c)

Fig 4. Comparison of the results obtained from the proposed method and state-of-the-art methods when applied on; (a) MTDB, (b)

ITESOFT, (c) CLEF-IP datasets.

\section{CONCLUSION}

In the present study, an efficient document image retrieval method based on human visual system/attention is presented. Visual saliency maps of document images were obtained and considered as weighting maps to emphasise the foreground pixels in the original images. Then, features were extracted from the weighted document images by employing the Gist descriptor. The proposed DIR method increased the retrieval results compared to the Gist operator. Due to the existing variation in structure and content of the documents, the DIR is still an open research problem. In our future work, we will consider saliency maps with deep learning-based methods for the DIR process.

\section{REFERENCES}

[1] Kazutaka Takeda, Koichi Kise, and M. Iwamura, "Real-Time Document Image Retrieval for a 10 Million Pages Database with a Memory Efficient and Stability Improved LLAH," in Proceedings of the ICDAR, pp. 1054-1058, 2011.
[2] JilinLi, Z.-G. Fan, Y. Wu, and N. Le, "Document Image Retrieval with Local Feature Sequences," in Proceedings of the ICDAR, pp. 346-350, 2009.

[3] A. Alaei, P. P. Roy, and U. Pal, "Logo and seal based administrative document image retrieval: a survey," in Computer Science Review, vol. 22, pp. 47-63, 2016.

[4] H. Gao, M. Rusinol, D. Karatzas, J. Lladós, T. Sato, M. Iwamura, et al., "Key-Region Detection for Document Images-Application to Administrative Document Retrieval," in Proceedings of the ICDAR, pp. 230-234, 2013

[5] J. Kumar, P. Ye, and D. Doermann, "Structural similarity for document image classification and retrieval," in Pattern Recognition Letters, vol. 43, pp. 119-126, 2014.

[6] Guangyu Zhu and David Doermann, "Logo matching for document image retrieval," in Proceedings of the ICDAR, pp. 606-610, 2009.

[7] R. C. Gonzalez and R. E. Woods, "Book on "Digital Image Processing"," ed: Prentice-Hall of India Pvt. Ltd, 2005.

[8] S. G. Mallat, "A theory for multiresolution signal decomposition: the wavelet representation," in PAMI, vol. 11, pp. 674-693, 1989.

[9] M. Douze, H. Jégou, H. Sandhawalia, L. Amsaleg, and C. Schmid, "Evaluation of gist descriptors for web-scale image search," in Proceedings of the ACM, pp. 19-27, 2009.

[10] F. Alaei, A. Alaei, U. Pal, and M. Blumenstein, "Document Image Retrieval Based on Texture Features: A Recognition-Free Approach," in In Proceedings of the DICTA, pp. 1-7, 2016

[11] F. Alaei, A. Alaei, M. Blumenstein, and U. Pal, "Document image retrieval based on texture features and similarity fusion," in In Proceedings of the IVCNZ, pp. 1-6, 2016.

[12] A. Haar, "On the theory of orthogonal function systems," in MATH ANN, vol. 69, pp. 331-371, 1910 .

[13] M. Singha, "Content Based Image Retrieval using Color and Texture," in SIPIJ, vol. 3, pp. 39-57, 2012.

[14] A. Oliva and A. Torralba, "Modeling the shape of the scene: A holistic representation of the spatial envelope," in CVIP, vol. 42, pp. 145-175, 2001.

[15] A. Oliva and A. Torralba, "Building the gist of a scene: The role of global image features in recognition," Progress in brain research, vol. 155 , pp. 23-36, 2006

[16] Z. Li and L. Itti, "Saliency and gist features for target detection in satellite images," in Image Processing, vol. 20, pp. 2017-2029, 2011.

[17] W. Liu, S. Kiranyaz, and M. Gabbouj, "Robust scene classification by gist with angular radial partitioning," in ISCCSP, pp. 1-6, 2012.

[18] Y. Gao, M. Shi, D. Tao, and C. Xu, "Database saliency for fast image retrieval,"in Multimedia, vol. 17, pp. 359-369, 2015.

[19] C. Bai, J.-n. Chen, L. Huang, K. Kpalma, and S. Chen, "Saliency-based multi-feature modeling for semantic image retrieval," in JVCIR, vol. 50, pp. 199-204, 2018

[20] J. Li, M. D. Levine, X. An, X. Xu, and H. He, "Visual saliency based on scale-space analysis in the frequency domain," in PAMI, vol. 35 , pp. 996-1010, 2013.

[21] M. Jian, L. Wu, C. Jung, Q. Fu, and T. Jia, "Visual saliency estimation using constraints," in Neurocomputing, vol. 290, pp. 1-11, 2018.

[22] F. Battisti, S. Baldoni, M. Brizzi, and M. Carli, "A feature-based approach for saliency estimation of omni-directional images," in SPIC, vol. 69 , pp. 53-59, 2018

[23] F. Alaei, A. Alaei, U. Pal, and M. Blumenstein, "Evaluation of Gist Operator for Document Image Retrieval," in Proceedings of the DAS, , pp. 369-374, 2018.

[24] F. Alaei, A. Alaei, U. Pal, and M. Blumenstein, "A Comparative Study of Different Texture Features for Document Image Retrieval," in ESWA, vol. 121, pp.97-114, 2019

[25] C. Zhao, C. Liu, and Z. Lai, "Multi-scale gist feature manifold for building recognition," in Neurocomputing, vol. 74, pp. 2929-2940, 2011.

[26] Y. Zhou, C. Liu, N. Li, and M. Li, "A novel locality-sensitive hashing algorithm for similarity searches on large-scale hyperspectral data," in RSL, vol. 7, pp. 965-974, 2016.

[27] J. Sauvola and H. Kauniskangas, "MediaTeam document database II," A CD-ROM collection of document images, University of Oulu Finland, 1999.

[28] F. Alaei, N. Girard, S. Barrat, and J.-Y. Ramel, "A New One-Class Classification Method Based on Symbolic Representation: Application to Document Classification," in Proceedings of the DAS, pp. 272-276, 2014.

[29] F. Piroi, M. Lupu, A. Hanbury, and V. Zenz, "CLEF-IP 2011: Retrieval in the Intellectual Property Domain," in CLEF (notebook papers/labs/workshop), 2011 